\title{
Surface morphology and deuterium retention of tungsten after low- and high-flux deuterium plasma exposure
}

\author{
M.H.J. 't Hoen ${ }^{1}$, M. Balden'2, A. Manhard ${ }^{2}$, M. Mayer ${ }^{2}$, \\ S. Elgeti ${ }^{2}$, A.W. Kleyn ${ }^{1,3,4}$ and P.A. Zeijlmans van Emmichoven ${ }^{1}$ \\ ${ }^{1}$ FOM institute DIFFER, Association EURATOM-FOM, Partner in the Trilateral \\ Euregio Cluster, NL-3439 MN Nieuwegein, the Netherlands \\ ${ }^{2}$ Max-Planck-Institut für Plasmaphysik, Boltzmannstraße 2, D-85748 Garching, \\ Germany \\ ${ }^{3}$ Van 't Hoff Institute for Molecular Sciences, University of Amsterdam, Science Park \\ 904, NL-1098 XH Amsterdam, the Netherlands \\ ${ }^{4}$ Chengdu Development centre for Science and Technology, Shuangliu, Chengdu, \\ Sichuan 610207, People's Republic of China \\ E-mail: $m \cdot h \cdot j \cdot$ thoen@differ.nl
}

\begin{abstract}
.
The surface morphology and deuterium retention were investigated of polycrystalline tungsten targets that were exposed to deuterium plasmas at widely varying conditions. By changing only one parameter at a time, the isolated effects of flux, time and pre-damaging on surface modifications and deuterium retention were studied. The sample exposed to low-flux plasma $\left(10^{20} \mathrm{~m}^{-2} \mathrm{~s}^{-1}\right)$ is mostly smooth with only a few areas containing very large blisters $(50-500 \mu \mathrm{m})$. The samples exposed to high-flux plasmas $\left(10^{24} \mathrm{~m}^{-2} \mathrm{~s}^{-1}\right)$ show large numbers of smaller blisters $(1-10 \mu \mathrm{m})$ and in addition even smaller protrusions $(<750 \mathrm{~nm})$. The size of the blisters and their density strongly increase with fluence. Pre-damaging tungsten with $\mathrm{MeV}$ ions leads to less blisters but to more protrusions. In addition to these (sub-)micrometer-sized structures, all samples show formation of nanostructures. Comparison of a low-flux and high-flux sample exposed to similar fluence showed that the variation in morphology is dominated by the flux differences. It is shown that the blisters and protrusions originate in inter- and intra-granular cavities, respectively. The depth of the cavities underneath the surface correlates well with the depth distributions of the retained deuterium. Trapping of significant amounts of deuterium therefore seems to take place in and/or close to these cavities and gives rise to an additional peak in the thermal desorption spectrum at $700 \mathrm{~K}$.
\end{abstract}

PACS numbers: 28.52.Fa, 28.52.Nh, 52.40.Hf, 52.77.Dq, 61.72.-y, 61.80.Jh, 68.37.-d

Submitted to: Nuclear Fusion 


\section{Introduction}

It has recently been decided that tungsten will be used in the divertor of the experimental fusion reactor ITER from the beginning of operation [1]. The divertor serves as the exhaust of the tokamak where the helium ash is removed. It is in that particular region that the most intense interactions between the plasma and the plasma-facing components take place. The partially detached plasma in the divertor region are expected to have a high electron density $\left(10^{20}-10^{21} \mathrm{~m}^{-3}\right)$ and electron temperatures of $1-10 \mathrm{eV}$ [2, 3]. This leads to extremely intense particle and heat flux densities on the divertor of $\sim 10^{24}$ ions $\mathrm{m}^{-2} \mathrm{~s}^{-1}$ and $\sim 10 \mathrm{MW} \mathrm{m}^{-2}$, respectively. Its high thermal conductivity and melting point as well as its low erosion rate make tungsten favourable over many other materials. It is in addition well known that the uptake of hydrogen by tungsten is low, which is important since, for safety and efficiency reasons, the total tritium inventory in ITER should be kept below $700 \mathrm{~g}$ [4].

Even though the physical properties of tungsten seem to be quite good, it has been shown in several experiments that the extreme conditions as expected in part of the ITER divertor may lead to severe modifications of tungsten [5, 6, 7, 8, 9]. This also occurs when the incident energy is far below the threshold for displacement damage and is strongly dependent on the specific tungsten material structure. Blister formation originating from growth of inter-granular voids is one example that has been studied extensively [10, 11]. Although this type of material (rolled tungsten) will not be be used for the ITER divertor, investigation of such materials are necessary to improve understanding of the blister formation mechanism. Surface morphology changes may lead to degradation of material properties as well as to enhanced erosion and formation of dust. It has also been shown that surface modifications can lead to enhanced hydrogen retention [12, 13]. In several experiments it has furthermore been shown that the retention will be significantly enhanced by radiation damage due to the bombardment with neutrons or with MeV particles [14, 15, 16, 17].

In this paper we study the effect of flux, fluence and pre-irradiation damage on surface modifications and deuterium retention. We will show that the sizes of the surface modifications range from the $\mathrm{nm}$ to the $\mu \mathrm{m}$ scale and that the depth distributions of trapped deuterium correlate with damage in the material. It will also be shown that experiments carried out at similar fluence but at different flux show large differences in surface modifications as well as in deuterium retention.

\section{Experiment}

Four polycrystalline tungsten targets were cut from a rolled sheet $(0.9 \mathrm{~mm}$ thick, PLANSEE, 99.96\% purity) [18]. They were mechanically polished until the surface was mirror-like, so that changes in surface morphology could easily be studied. Subsequently, the samples were ultrasonically cleaned in isopropanol and heated for $1 \mathrm{~h}$ at $1200 \mathrm{~K}$ in vacuum $\left(<10^{-6} \mathrm{~Pa}\right)$ to relieve material stresses, for hydrogen degassing and oxide layer 
removal. One of the samples was pre-irradiated with $12.3 \mathrm{MeV} \mathrm{W}^{4+}$ ions to a maximum damage level of $0.45 \mathrm{dpa}$ (displacements per atom) to simulate the neutron damage that is expected in ITER. The dpa level was calculated using SRIM [19], assuming a displacement energy $\left(\mathrm{E}_{\text {displacement }}\right)$ of $90 \mathrm{eV}$ [20]. The $3 \mathrm{MV}$ tandem ion accelerator at IPP-Garching irradiated an area of $18 \mathrm{~mm}$ in diameter.

\subsection{Deuterium plasma exposure}

The tungsten targets were exposed in two devices, PlaQ [21] and Pilot-PSI [22]. PlaQ, located at IPP-Garching, produces plasma fluxes of about $10^{20} \mathrm{~m}^{-2} \mathrm{~s}^{-1}$. The plasma ions mainly consist of $\mathrm{D}_{3}^{+}$with an ion energy of $38 \mathrm{eV}$ per atom [21]. The exposure is homogeneous across the rectangular specimen $(12 \times 15 \mathrm{~mm})$. The target was heated with a boralectric heater to a surface temperature of $500 \mathrm{~K}$. After plasma exposure and simultaneously switching off the heater, cooling is relatively slow: the first $50 \mathrm{~K}$ typically takes about 5 minutes, 30 minutes are needed to reach $320 \mathrm{~K}$.

Pilot-PSI, which is located at FOM-DIFFER, produces $\mathrm{D}^{+}$particle fluxes of typically $10^{24} \mathrm{~m}^{-2} \mathrm{~s}^{-1}$ in a magnetic field of $0.4 \mathrm{~T}$. A bias voltage of $-40 \mathrm{~V}$ was applied to the targets $(\varnothing 20 \mathrm{~mm})$. The plasma potential is not precisely known [23, 24] but is only a few eV. The ion energy is therefore dominated by the target bias and close to $40 \mathrm{eV}$. The electron density and temperature of the Pilot-plasma were measured with Thomson scattering [25]. This measurement was used to estimate the ion flux on the

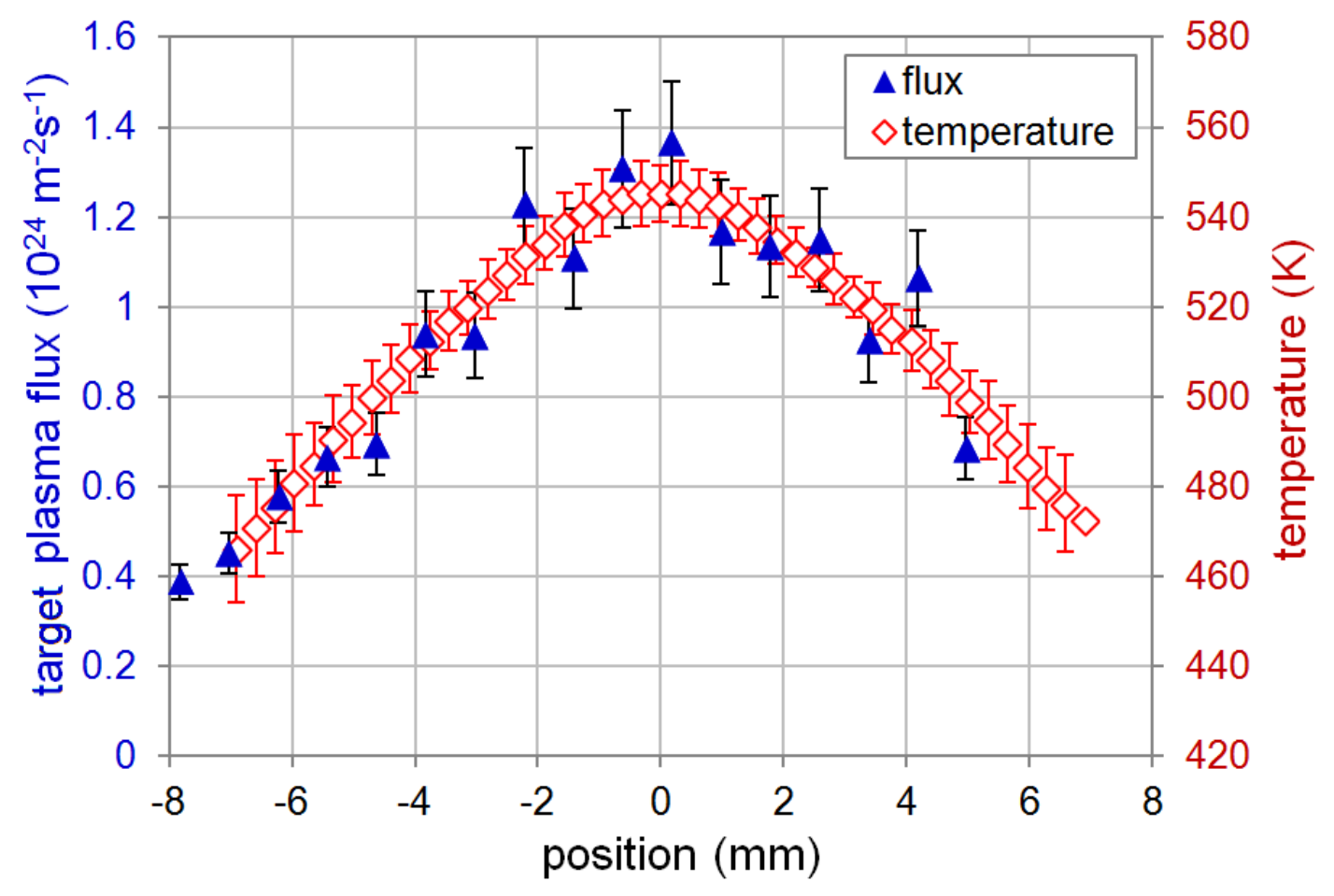

Figure 1. Radial dependence of the plasma flux (solid triangles) and surface temperature (open diamonds) at the target during plasma exposure of sample D. 


\begin{tabular}{lllll}
\hline & $\begin{array}{l}\text { sample A } \\
\text { low-flux }\end{array}$ & $\begin{array}{l}\text { sample B } \\
\text { low-fluence }\end{array}$ & $\begin{array}{l}\text { sample C } \\
\text { high-fluence }\end{array}$ & $\begin{array}{l}\text { sample D } \\
\text { pre-damaged }\end{array}$ \\
\hline pre-irradiation & - & - & - & $0.45 \mathrm{dpa}$ \\
& & & $12.3 \mathrm{MeV} \mathrm{W}^{4+}$ \\
flux $(\Gamma)$ & $10^{20} \mathrm{~m}^{-2} \mathrm{~s}^{-1}$ & $1.1 \times 10^{24} \mathrm{~m}^{-2} \mathrm{~s}^{-1}$ & $1.0 \times 10^{24} \mathrm{~m}^{-2} \mathrm{~s}^{-1}$ & $1.1 \times 10^{24} \mathrm{~m}^{-2} \mathrm{~s}^{-1}$ \\
fluence $(F)$ & $10^{26} \mathrm{~m}^{-2}$ & $0.8 \times 10^{26} \mathrm{~m}^{-2}$ & $0.8 \times 10^{27} \mathrm{~m}^{-2}$ & $0.8 \times 10^{27} \mathrm{~m}^{-2}$ \\
temperature & $500 \mathrm{~K}$ & $515 \mathrm{~K}$ & $530 \mathrm{~K}$ & $530 \mathrm{~K}$ \\
ion energy & $38 \mathrm{eV} / \mathrm{D}$ & $\sim 40 \mathrm{eV} / \mathrm{D}$ & $\sim 40 \mathrm{eV} / \mathrm{D}$ & $\sim 40 \mathrm{eV} / \mathrm{D}$ \\
\hline
\end{tabular}

Table 1. Overview of the exposure conditions of the four investigated samples. The plasma conditions and surface temperature of sample A are constant over the whole sample. Plasma exposure at Pilot-PSI (samples B - D) results in an inhomogeneous surface temperature profile (figure 1). The temperature mentioned for samples B - D is given at $2 \mathrm{~mm}$ from the centre, where the SEM investigations and NRA measurements were performed.

target by applying the Bohm criterion [26]. In figure 1 the radial distribution of the flux is shown. The Gaussian flux profile is the result from the measured Gaussian electron density profile and the plasma temperature profile. The plasma beam heats the target, therefore the temperature profile resembles the flux distribution (figure 1). At the start of each plasma shot, it typically takes $1-2$ seconds before the sample reaches its steadystate temperature. The temperature profile of the tungsten surface during exposure was measured with a fast infrared camera (FLIR SC7500-MB) and ranges between $545 \mathrm{~K}$ in the centre of the target to $460 \mathrm{~K}$ at the edges (the emissivity of the tungsten targets used was 0.06, a value verified ex situ with a thermocouple measurement). During plasma exposure, the targets were water-cooled via a copper block. The cooling of these targets is very effective, after plasma exposure the sample cooled down to room temperature typically within a few seconds.

The four samples were exposed to plasma under very different conditions. From one sample to the next, however, only one parameter was varied whereas all the others were kept as close as possible. In this way we are able to study the isolated effects of plasma flux, plasma fluence (time) and pre-irradiation damage on surface modifications and deuterium retention.

An overview of the main parameters as used in the experiments is given in table 1. Sample A, which we call the low-flux sample, was exposed in PlaQ [27]. The other samples $(B-D)$ were exposed to high-flux deuterium plasmas in Pilot-PSI. The surface temperatures mentioned for samples $\mathrm{B}-\mathrm{D}$ are the values at $2 \mathrm{~mm}$ from the centre of the spot on the round sample, where the surface morphology and the deuterium retention were studied. The main difference between the exposure conditions of samples A and $\mathrm{B}$ was the flux that varied by four orders of magnitude. Also the deuterium plasma fluence was kept close, i.e. the samples were exposed to similar amounts of particles. In order to reach the particle fluence of $10^{26} \mathrm{~m}^{-2}$ in PlaQ, the sample was exposed for 
about 12 days. Sample B, which we will call the low-fluence sample, reached a similar particle fluence already after seven shots of $10 \mathrm{~s}$ in Pilot-PSI. Sample C, which we will call the high-fluence sample, was exposed to ten plasma shots of $75 \mathrm{~s}$, so that a fluence of $0.8 \times 10^{27} \mathrm{~m}^{-2}$ was reached. The major difference between the exposures of the lowfluence and high-fluence samples is the exposure time. In this way, the effect of exposure time during high-flux plasma exposure was studied. Sample D is the sample that was pre-irradiated to a damage level of $0.45 \mathrm{dpa}$ prior to plasma exposure and is called the pre-damaged sample. All plasma exposure conditions of sample $\mathrm{D}$ were very close to those of sample $\mathrm{C}$.

\subsection{Target analysis}

After deuterium implantation, all four samples were investigated to study surface morphology and deuterium retention. Surface modifications were studied with optical and scanning electron microscopy (SEM). As will be discussed, blisters of different sizes were observed. Several blisters were cut with a focussed ion beam (FIB) to determine the bulk structure beneath them. Subsequently, the samples were investigated by nuclear reaction analysis (NRA) to study the deuterium depth distributions. Thermal desorption spectroscopy (TDS) was used to get information about the binding energies of the deuterium retained in tungsten.

Scanning electron microscopy images were acquired with a Helios NanoLab600 (FEI) at IPP-Garching. Images were typically taken using a $5 \mathrm{keV}$ electron beam. The surface of the low-flux target (A) was also analysed with a confocal laser scanning microscope (CLSM).

Information about the region underneath the blisters was obtained by combining SEM imaging with focused ion beam cutting. The Ga-ion beam of the Helios NanoLab600 with an energy of $30 \mathrm{keV}$ and current between 2 and $20 \mathrm{nA}$ was used to create cross-sections. To reduce artefacts due to the cutting process, the area of interest was coated with a Pt-C film prior to the cutting. The surface normal was aligned with the ion beam. The angle between Ga-ion and electron beam was $52^{\circ}$. Therefore, the cross-sections are imaged under $38^{\circ}$, causing a difference between the horizontal and vertical scale. The length of the vertical scale bars in the images are therefore 0.79 of the horizontal scale bars.

Nuclear reaction analysis of the targets exposed in Pilot-PSI was performed two weeks after plasma exposure at IPP-Garching. The nuclear reaction $\mathrm{D}\left({ }^{3} \mathrm{He}, \mathrm{p}\right)^{4} \mathrm{He}$ [28, 29] was used to obtain a local measurement of the deuterium concentration as function of depth. The ${ }^{3} \mathrm{He}$ beam spot of $1 \mathrm{~mm}^{2}$ size was positioned for the samples B - D $2 \mathrm{~mm}$ off-centre of the Pilot-PSI exposure spot. The energy was varied in 6 steps from $690 \mathrm{keV}$ to $4.0 \mathrm{MeV}$ to determine the deuterium concentration down to a depth of $6 \mu \mathrm{m}$. The low-flux sample (A) was analysed using slightly different energies [27]. The depth profiles of retained deuterium were calculated from the measured proton energy distributions by use of the NRA-DC program [30]. 
The targets were analysed with thermal desorption spectroscopy at FOM-DIFFER 5 months after Pilot-PSI plasma exposure. The tungsten targets were clamped to a ceramic heater and heated with a linear temperature ramp of $1 \mathrm{~K} / \mathrm{s}$ to $1273 \mathrm{~K}$ and held for $10 \mathrm{~min}$ at $1273 \mathrm{~K}$. A Balzers QMA125 quadrupole mass spectrometer monitored the mass $4\left(D_{2}\right)$ signals in the residual gas in the chamber. To determine the total amount of deuterium released from the target during the temperature ramp, the absolute sensitivity was determined using a calibrated $\mathrm{D}_{2}$ leak. The mass 3 (HD) signal was in all cases less than $20 \%$ of the mass 4 signal.

\section{Results}

\subsection{Surface morphology}

CLSM and SEM images of the four investigated samples are shown in figure 2, The four images taken at the lowest magnification show the $100-\mu \mathrm{m}$ range with large blisters covering several grains. At higher magnification, individual grains become visible that contain smaller blisters for three of the targets. At the largest magnification, it is clear that all four targets contain structures on the nanometer scale.

On the low-flux sample (A), only few (about 60 on the surface area of about $1.7 \mathrm{~cm}^{2}$ ) but very large blisters $(50-500 \mu \mathrm{m})$ and some smaller micrometer-sized blisters were observed. The blisters are irregularly distributed over the sample surface and seem to appear in groups. One such region with three of these blisters is shown in figure $2 \mathrm{~A}$. Note that this image is taken with a confocal laser scanning microscope and that the scale differs from the other images. The surface of the area shown is smooth except for the blisters. In addition, a few micrometer-sized blisters $(1-10 \mu \mathrm{m})$ are observed. These smaller blisters are predominantly found in the neighbourhood and on top of the large blisters. The high-resolution SEM images mostly show a smooth surface on the micrometer scale and a nanostructured surface on the sub-micrometer scale. The type of nanostructure changes at the grain boundaries, indicating that the structure depends on the grain orientation. Stripe-like surface structures are observed as well as more randomly shaped structures.

The (high-flux) low-fluence sample is shown in figure $2 \mathrm{~B}$. It is covered by large amounts of blisters of only a few micrometer in diameter. These micrometer-sized blisters often extend over more than one grain. Zooming in at larger magnification reveals, in addition to the blisters, irregularly shaped features in the sub- $\mu \mathrm{m}$ range. We will call these $100-750 \mathrm{~nm}$ large features protrusions. Most of the protrusions appear within grains whereas their density strongly varies from one grain to the other. Zooming in even further reveals a nanostructured surface. Densely packed, randomly shaped structures with a size of $10-20 \mathrm{~nm}$ cover the surface. It should be noted that at locations not shown in figure $2 \mathrm{~B}$ also weak stripe-like structures appear. The appearance varies again from grain to grain.

The surface morphology of the high-fluence sample is shown in figure $2 \mathrm{C}$. Both 


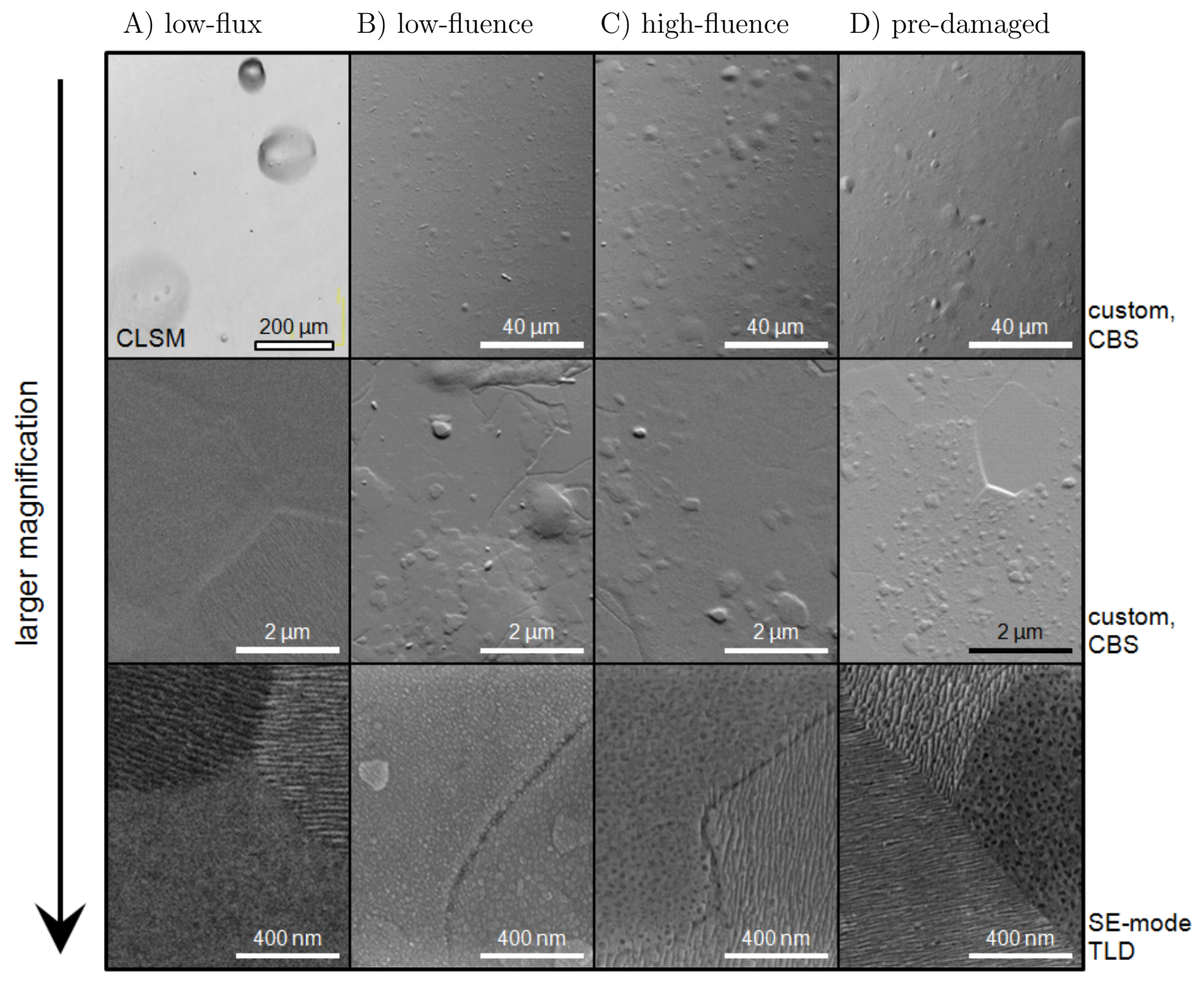

Figure 2. CLSM and SEM images of the four samples. From left to right the (A) low-flux, (B) low-fluence, (C) high-fluence and (D) pre-damaged sample. The SEM images were taken at three magnifications to show the $100-\mu \mathrm{m}$ range, the $\mu \mathrm{m}$ range and the sub- $\mu \mathrm{m}$ range. The lowest magnification of the low-flux sample (A) is the only one taken with a confocal laser scanning microscope, its scale differs from the other images. The low magnification SEM images are taken in custom mode with the concentric backscatter electron detector (CBS). The highest magnification image is taken using secondary electron (SE) mode with the tube lens detector (TLD).

the amount of blisters as well as their size has increased compared to the low-fluence sample. Zooming in reveals similar protrusions as observed on the low-fluence sample. It is not possible to determine whether their amount has increased or decreased, because of their irregular distribution and because they are only visible at a large magnification. Pronounced nanostructures are observed for the largest magnification, their detailed appearance being clearly correlated to the specific grains and therefore probably to grain orientation.

In figure 2D the SEM images for the pre-damaged sample are shown. Comparing to 
figure $2 \mathrm{C}$ shows that the amount of micrometer-sized blisters has decreased. This is the case also for other areas on the surface. Zooming in reveals a lot of small protrusions $(<250 \mathrm{~nm})$ formed within specific grains. The highest magnification shows again the appearance of nanostructures.

\subsection{Sub-surface morphology}

A focussed ion beam in combination with a SEM was used to create cross-sectional images of the blisters. A typical cross-section of one of the large blisters on the low-flux sample (A) is shown in figure 3. A large cavity is visible below the blister. As discussed in [10, 11, 31, 32], the solubility of hydrogen in tungsten is very low. This promotes the accumulation of deuterium gas in cavities. The pressure that builds up is a driving force for cavity growth. Material can be pushed upwards and deform the surface: a blister is formed. The cavity associated with the blister in figure 3 a appears at a depth of about $5 \mu \mathrm{m}$. Cutting other blisters revealed cavities at even larger depths up to $30 \mu \mathrm{m}$. The cracks follow the grain boundaries (figure 3e). Furthermore, the blisters did not collapse during the FIB cutting, which means that they are stable and were formed in a plastic deformation process [10].

The smaller micrometer-sized blisters that predominantly show up in the high-flux samples were also studied with the FIB in combination with SEM (figure 4). These blisters originate in cavities at grain boundaries as well and can extend over several grains. The cavities of the low-fluence sample (B) are typically observed up to about $1 \mu \mathrm{m}$ below the surface (figure 4 a). Cavities for the high-fluence sample (C) typically extend deeper in the material and are observed up to $2 \mu \mathrm{m}$ depth. It is also clear that the cavities are significantly larger in volume than the ones in the low-fluence sample. In figure 4 also a few cavities are shown that appear within a grain. These cavities are a few hundred nanometer in size and occur close to the sample surface. As result of this cavity formation, the before mentioned protrusions appear at the surface.
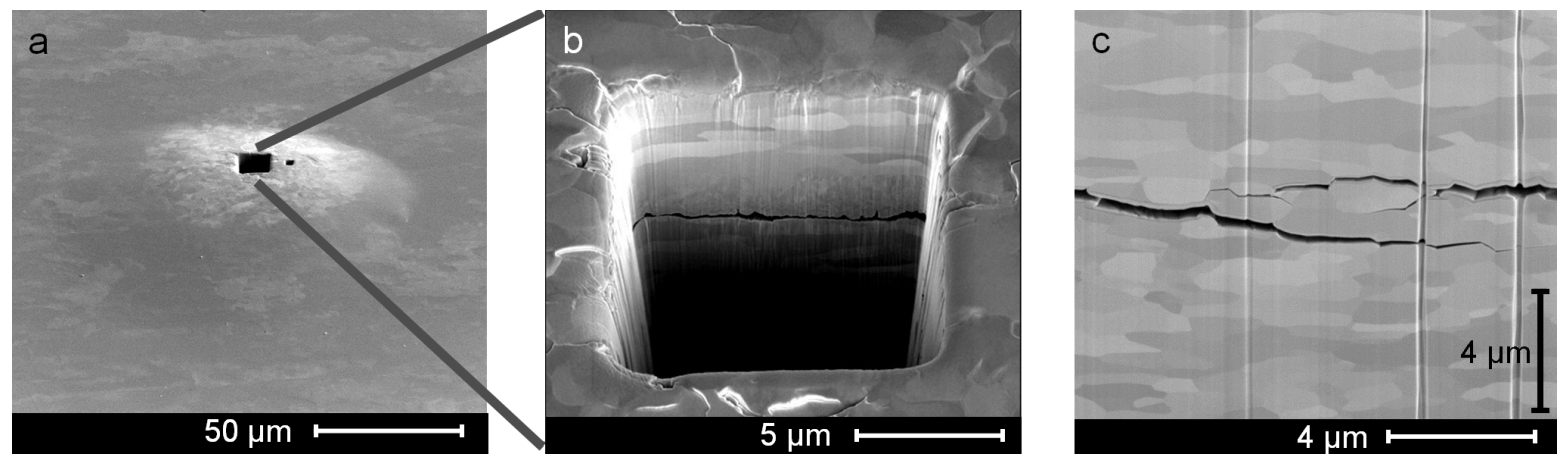

Figure 3. a) Large blister on the low-flux sample is cut by FIB. b) The associated cavity is found about $5 \mu \mathrm{m}$ below the surface (the cavity is partly filled during the FIB cutting by redeposited material). c) FIB cross-section of another cavity, where the cracks follows the grain boundaries. 

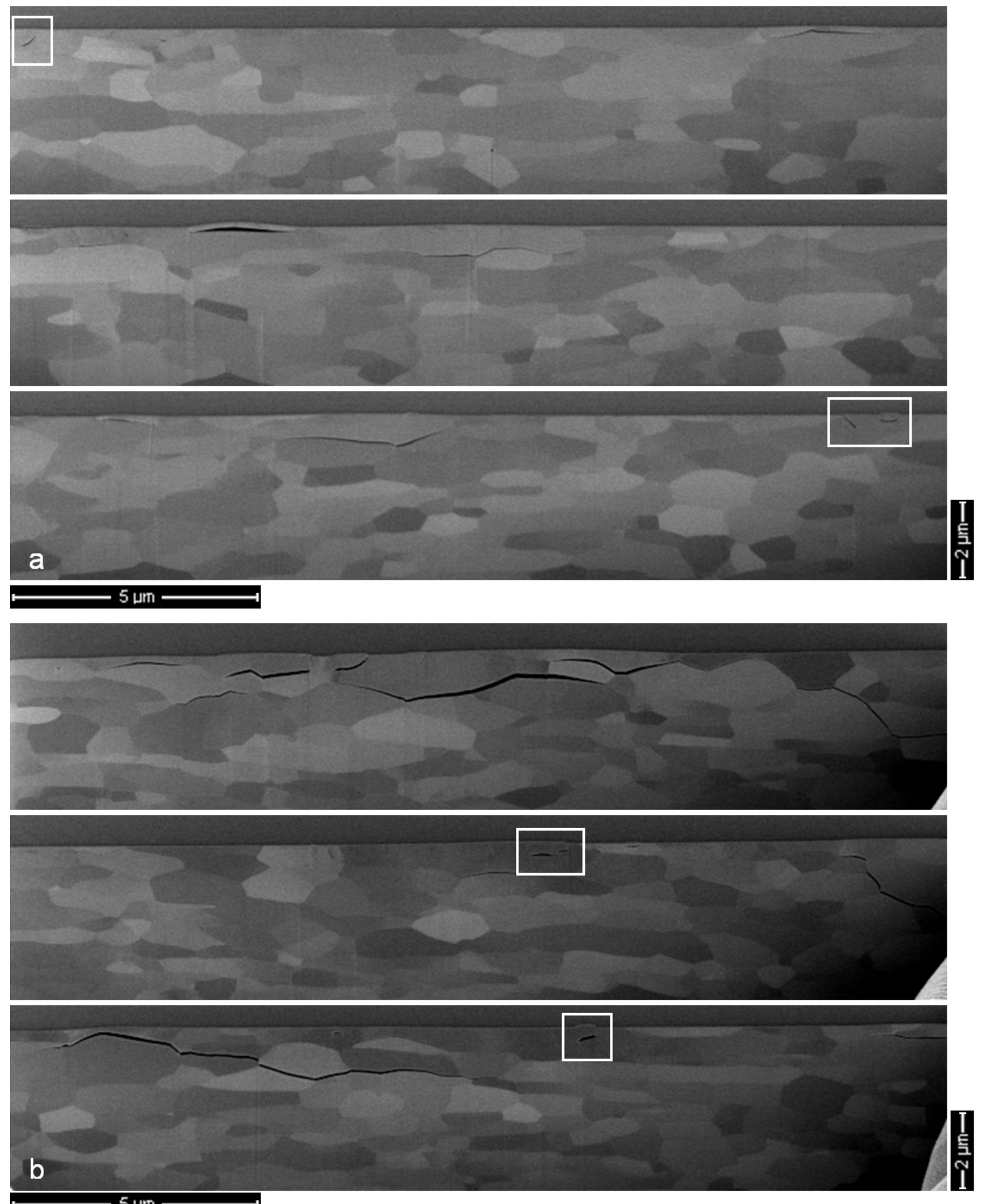

Figure 4. SEM images of the FIB cuts of the a) low-fluence (B) and b) high-fluence (C) sample. The cavities that appear within a grain are indicated with a white box.

In figure 5 a cross-sectional view of the pre-damaged sample (D) is shown. The image clearly shows the difference between a micrometer-sized blister and the smaller protrusions. The blister is associated with a cavity that arises along grain boundaries 


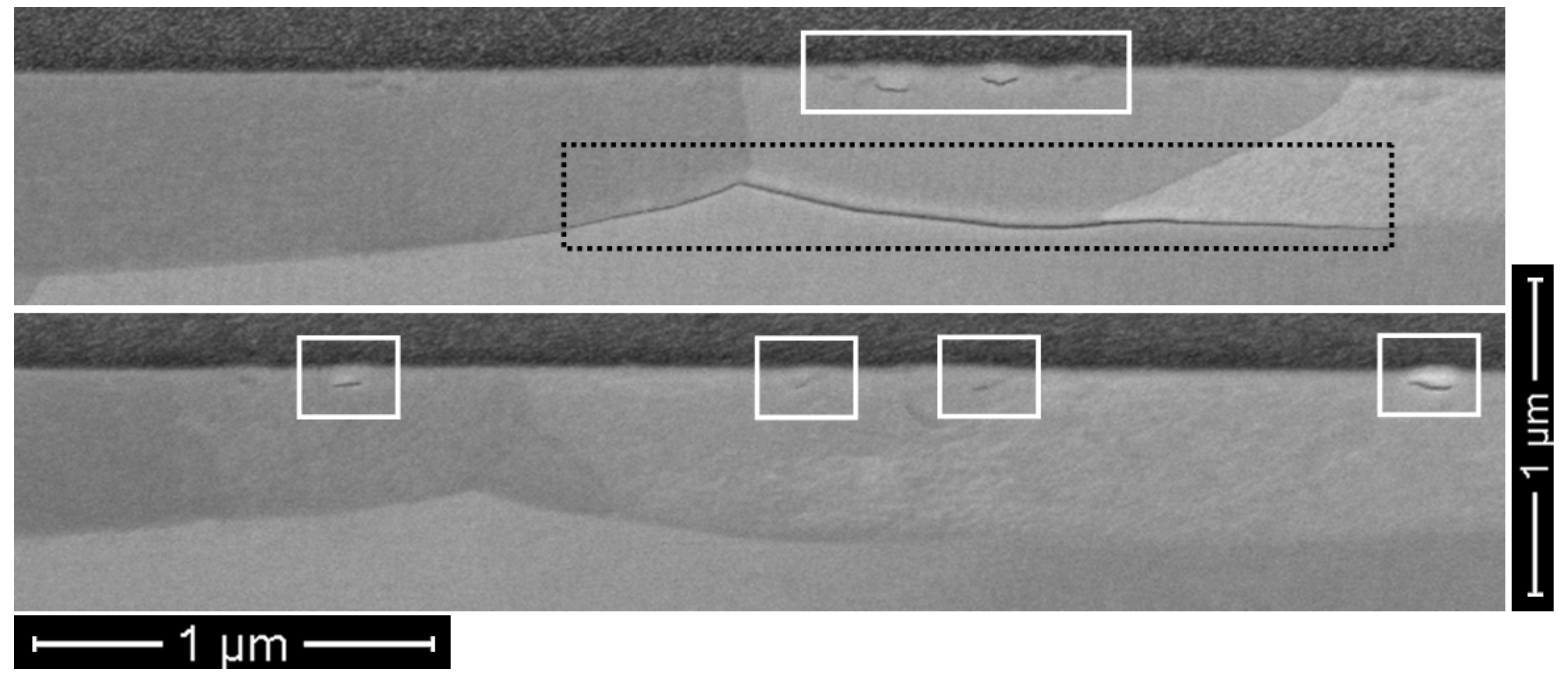

Figure 5. SEM images of cross-sections of the pre-damaged sample showing both an inter-granular cavity (black dotted rectangle) as well as intra-granular cracks (white rectangles)

about parallel to the surface (indicated by the dotted box). It is difficult to recognize this blister at the surface since its width is large compared to its height. The protrusions (indicated by the solid boxes) are associated with cracks within a grain. These cracks are located much closer to the surface at less than $100 \mathrm{~nm}$ depth. Deeper in the grain no cracks have been observed. In conclusion, the blisters originate in inter-granular cavities, protrusions arise from intra-granular cavities.

\subsection{Deuterium depth profiling}

In figure 6, the NRA depth profiles of the retained deuterium are shown. Whereas at the surface the deuterium concentration for all four targets is similar and relatively high, in the bulk significant differences are observed. The most likely deuterium depth profile of the low-flux sample (A) contains two layers. A sharp surface concentration and a constant deuterium concentration of about $10^{-4}$ atomic fraction (at.fr.) over the first $12 \mu \mathrm{m}$. This result follows from fitting the measured proton energy distributions with the NRA-DC program [30]. This constant concentration is in the same range as reported values for deuterium trapped in pre-existing defects [33], which depends on the tungsten grade. Note that this concentration is below the other three curves up to depths of $2-3 \mu \mathrm{m}$. Beyond this depth, the concentrations of the other samples are still decreasing and probably decrease below the fraction of the low-flux sample. Obviously, the exposure time of 12 days for the low-flux sample allowed the deuterium atom flux to penetrate deeper into the material as compared to the high-flux samples.

For the low-fluence sample (B), the deuterium concentration is highest at the surface, followed by a dip at $\sim 200 \mathrm{~nm}$. A local maximum in the deuterium concentration is observed at $0.5 \mu \mathrm{m}$, after which the concentration slowly decreases. Investigation of 


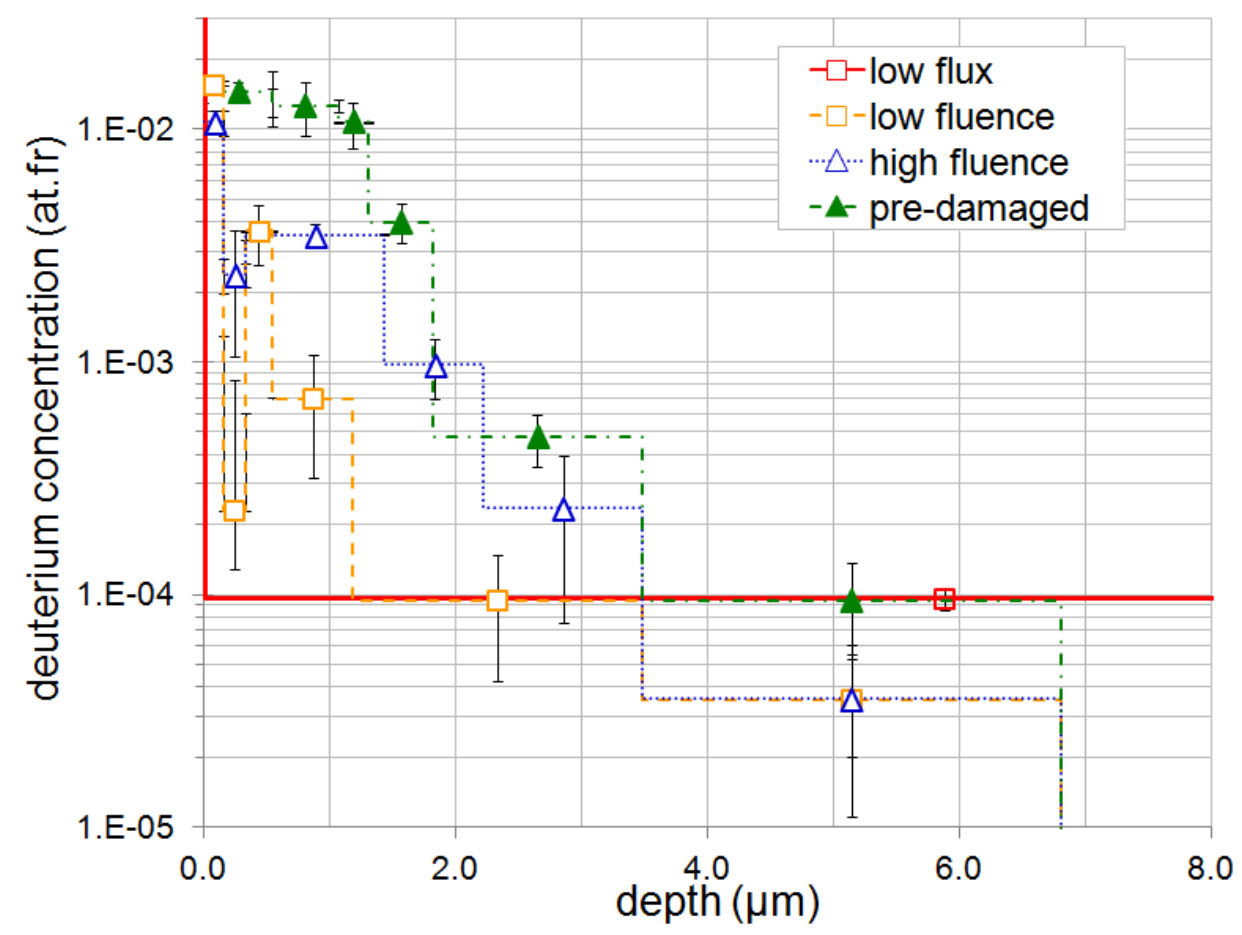

Figure 6. Deuterium depth profiles of the four samples. The squares indicate the samples exposed to low-fluence $\left(10^{26} \mathrm{~m}^{-2}\right)$ plasmas, the triangles the ones to a higher fluence of $10^{27} \mathrm{~m}^{-2}$. The low-flux sample $\left(\mathrm{A}, 10^{20} \mathrm{~m}^{-2} \mathrm{~s}^{-1}\right.$, PlaQ) is plotted with the thick red solid line, the low-fluence sample (B, $10^{24} \mathrm{~m}^{-2} \mathrm{~s}^{-1}$, Pilot-PSI) with the orange dashed line. The dotted blue line with open triangles represents the high-fluence sample (C), the green dash-dotted line with solid triangles the pre-damaged sample (D).

the high-fluence sample $(\mathrm{C})$ shows similar behaviour as the low-fluence sample. A small dip in the concentration just below the surface is followed by a local maximum at $0.7 \mu \mathrm{m}$ and then the concentration decreases slowly. The difference to the low-fluence sample is that the deuterium has penetrated deeper in the material. This corresponds well with the FIB results, where the cavities were found deeper in the material (figure 4).

The pre-damaged sample (D), finally, contains the largest concentration of retained deuterium, i.e. $1.2-1.4 \times 10^{-2}$ at.fr. in the first micrometer. This is consistent with our previous findings where it was shown that the deuterium is trapped predominantly in single vacancies, small vacancy clusters and dislocations [17]. It will be shown later in relation with the thermal desorption spectroscopy results, that part of the deuterium retention is related to the blister/ protrusion formation.

Integration of the deuterium concentration over the depth yields the total deuterium retained in the first $6 \mu \mathrm{m}$ (for the low-flux sample this is the first $12 \mu \mathrm{m}$ for as result of the difference in chosen NRA energies). For the low-flux, low-fluence, high-fluence and pre-damaged samples, the total deuterium retention at the investigated positions is 0.7 , $2.5,4.5$ and $12.8 \times 10^{20} \mathrm{~m}^{-2}$, respectively. 


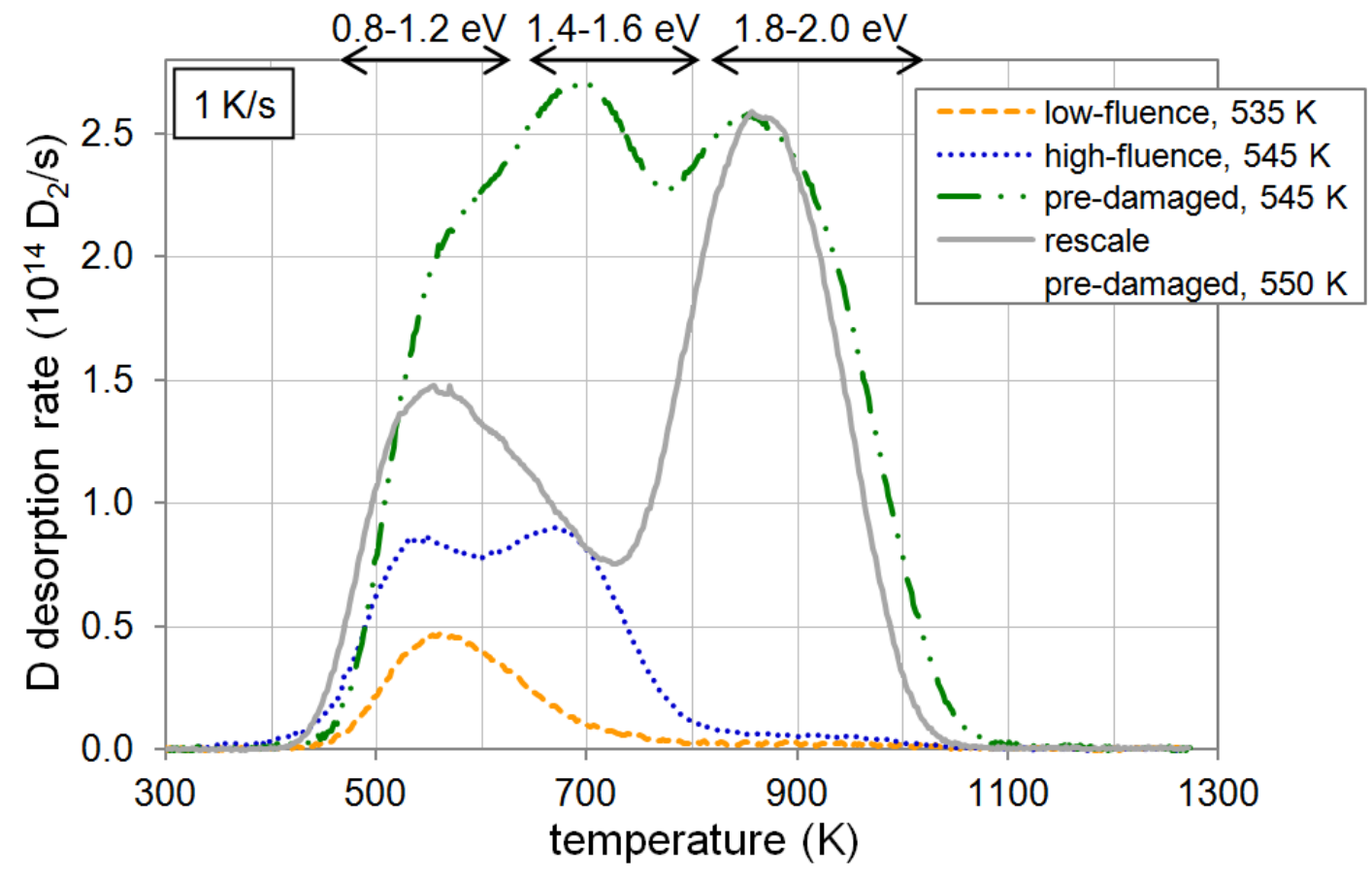

Figure 7. Thermal desorption spectra of the experiments carried out at high-flux (Pilot-PSI). The heating rate was $1 \mathrm{~K} / \mathrm{s}$. The low-fluence sample (B) is shown by the orange-dashed line, the high-fluence sample $(\mathrm{C})$ by the blue dotted line, and the predamaged sample (D) by the green dash-dotted line. The TDS spectrum of a previous pre-damaged sample (from [17]) is shown by the solid grey line. The latter spectrum was rescaled by matching the peak at about $850 \mathrm{~K}$ to the corresponding peak of the present pre-damaged sample. At the top of the image, the ranges of trapping energies that correspond to the position of the peaks are shown, in the case that the deuterium is retained as block profile in the first $1-2 \mu \mathrm{m}$.

\subsection{Deuterium desorption}

The TDS results for the three experiments carried out at high-flux are shown in figure 7. For comparison, also the TDS spectrum of a pre-damaged unpolished target is shown that was exposed for $100 \mathrm{~s}$ to high-flux plasma at self-biased conditions [17], at a surface temperature of $550 \mathrm{~K}$ in the centre of the exposure spot. The latter target did not show any surface blistering, although it should be mentioned that blisters are difficult to observe on a rough surface. Similar observations were made by Nishijima et al [34]. The desorption spectrum was rescaled by matching the peak at about $850 \mathrm{~K}$ to the corresponding peak of the present pre-damaged target, so that the ratio of the desorption peaks can easily be compared.

In figure 7, three clear desorption peaks can be distinguished located at about $550 \mathrm{~K}, 700 \mathrm{~K}$ and $850 \mathrm{~K}$. The low-fluence sample (B), with the least plasma damage of the high-flux samples, shows a desorption peak at $550 \mathrm{~K}$ and a small shoulder at higher energies. In the desorption spectrum of the high-fluence sample (C), with significantly more plasma damage, two peaks are present: at $550 \mathrm{~K}$ and at $700 \mathrm{~K}$. The pre-damaged 
sample (D), finally, shows strongly increased retention and three desorption peaks: at 550, at 700 and at $850 \mathrm{~K}$. The rescaled pre-damaged sample from [17] shows two pronounced desorption peaks at $550 \mathrm{~K}$ and at $850 \mathrm{~K}$, and a small shoulder at $700 \mathrm{~K}$.

TMAP7 simulations [35] were used to estimate the peak positions in the TDS spectra as function of deuterium trap energy. In the simulations, block profiles of deuterium traps up to a depth of $1,1.5$ and $2 \mu \mathrm{m}$ were used to take into account the range of depths at which deuterium is trapped. The ranges of the deuterium trap energies are indicated above the curves.

The desorption peak at $550 \mathrm{~K}$ is observed in all spectra. Based on the trapping energy of $0.8-1.2 \mathrm{eV}$, its presence can be ascribed to trapping of deuterium at dislocations, grain boundaries and/or mono-vacancies [17. The peak is larger for the high-fluence sample as compared to the low-fluence sample. For the pre-damaged sample the peak seems to have increased even further.

The desorption peak at $700 \mathrm{~K}$ appears as a small shoulder in the low-fluence sample. In the high-fluence as well as in the pre-damaged sample this desorption peak is clearly distinguishable. These are the two targets where plasma induced blisters and small protrusions are most pronounced. The rescaled pre-damaged sample from [17, where no blistering is observed, does not show a pronounced desorption peak at $700 \mathrm{~K}$. This correlation suggests that the desorption peak at $700 \mathrm{~K}$ is related to the plasma-induced modifications and that these modifications trap deuterium at energies of $1.4-1.6 \mathrm{eV}$.

The $850 \mathrm{~K}$ desorption peak appears only for the pre-damaged targets and corresponds to trapping of deuterium at $1.8-2.0 \mathrm{eV}$. This peak was attributed to trapping of deuterium in small vacancy clusters that are caused by the irradiation with $\mathrm{MeV}$ heavy ions [36, 37].

\section{Discussion}

In the present experimental results, three different types of surface modifications have been distinguished (table 21): blisters, protrusions and nanostructures. Blisters are hemispherical structures in the $1-500 \mu \mathrm{m}$ range that appear on the surface and that are caused by cavities below the surface. These cavities are formed at grain boundaries and are thus inter-granular. The second feature, the protrusions, have sizes of tens to hundreds of nanometers. Protrusions have an irregular shape and much sharper edges than the blisters. Cross-sections with the FIB show that the associated cavities are intragranular (figure 5). Protrusions appear with a large number density on certain grains whereas on others they are completely absent. This is probably related to the grain orientation [38. The nanostructures, finally, typically have sizes of $10-40 \mathrm{~nm}$. These structures have been well characterized by $\mathrm{Xu}$ et al for high-flux plasma conditions: they correlated the spongy, lamellae and triangular structures to $\langle 001\rangle,\langle 011\rangle$ and $\langle 111\rangle$ grain orientations, respectively [38].

Formation of blisters has been studied extensively in the past [10, 31, 32]. Tungsten has a very low solubility for hydrogen. In case the interstitial deuterium concentration 


\begin{tabular}{|c|c|c|c|c|c|}
\hline \multicolumn{2}{|c|}{ type of surface morphology } & size & depth & shape & location \\
\hline \multirow{2}{*}{ blisters } & huge & $50-500 \mu \mathrm{m}$ & $5-30 \mu \mathrm{m}$ & \multirow{2}{*}{$\begin{array}{l}\text { circular/ oval } \\
\text { smooth }\end{array}$} & \multirow{2}{*}{$\begin{array}{l}\text { inter- } \\
\text { granular }\end{array}$} \\
\hline & micrometer-sized & $1-10 \mu \mathrm{m}$ & $<2 \mu \mathrm{m}$ & & \\
\hline \multirow{2}{*}{ protrusions } & normal & $<750 \mathrm{~nm}$ & \multirow{2}{*}{$<100 \mathrm{~nm}$} & \multirow{2}{*}{$\begin{array}{l}\text { irregular } \\
\text { sharp edges }\end{array}$} & \multirow{2}{*}{$\begin{array}{l}\text { intra- } \\
\text { granular }\end{array}$} \\
\hline & small & $<250 \mathrm{~nm}$ & & & \\
\hline \multicolumn{2}{|c|}{ nanostructures } & $10-40 \mathrm{~nm}$ & & various & (sub)surface \\
\hline
\end{tabular}

Table 2. Classification of the surface modifications.

exceeds the solubility, i.e. in case of supersaturation, deuterium starts to precipitate. Accumulation of deuterium is expected to happen at nucleation points, which can be defects like dislocations, grain boundaries and vacancies. Subsequently, cavity growth can take place. Condon [32] distinguished three mechanisms for cavity growth: plastic deformation, dislocation loop punching and growth by vacancy clustering. For the last mechanism, temperatures above the temperature where vacancies get mobile are needed $(\sim 550 \mathrm{~K})$. The surface temperatures during plasma exposure were below this temperature. Although there is a possibility that the presence of deuterium enhances the vacancy mobility [39, 40], it is not likely that cavity growth by vacancy clustering played a role. Plastic deformation as well as dislocation loop punching start with pressure build up inside a cavity. To cause plastic deformation of grains, pressures in the range of a few hundred MPa have been reported [27, for plastic deformation caused by dislocation loop punching a few GPa is needed [9, 41]. From our results we are not able to distinguish between these two mechanisms.

The low-flux sample (A) does only show blisters on a few locations on the surface. Most striking are the very large ones with the underlying cavities being present at large depths of $5-30 \mu \mathrm{m}$. The fact that most of the surface is free of blisters and that where the blisters appear they do so in groups, indicates that the material contains weak grain boundaries in specific areas at large depths. These very large blisters only appear on the low-flux sample, because the long exposure time (four orders of magnitude higher than the high-flux exposures) allows the deuterium to accumulate at these large depths (see figure 6).

The high-flux samples are covered with micrometer-sized blisters. They originate in cavities at grain boundaries. The blisters do not collapse during cutting with the FIB, which means that they are plastically deformed. The increase of the size of the micrometer-sized blisters with exposure time (high-fluence $(\mathrm{C}$ ) versus low-fluence $(\mathrm{B})$ target) is probably related to the pressure build up over time. The increased amount of blisters and corresponding cavities can be explained by deuterium diffusing to larger depths and thereby reaching more nucleation points. Less micrometer-sized blisters are formed on the pre-damaged sample (D). This was observed before in other experiments 
carried out at lower flux and fluence [42]. The reason may be the presence of large amounts of nucleation points inside the grains that are decorated with deuterium. Possibly, these decorated traps can adapt better to stresses accumulated at the grain boundaries because of hydrogen enhanced local plasticity [39, 40].

On the high-flux samples, we also observed the formation of protrusions with sizes up to $750 \mathrm{~nm}$. We have shown that they originate in cavities within grains. On the low-flux sample such protrusions were not observed. Obviously, the hydrogen interstitial concentration needs to be high enough to promote cavity formation within a grain. The formation probably starts at a nucleation point that is already present in the material before plasma exposure. This is consistent with the fact that in the pre-damaged target, with many vacancies and small vacancy clusters present, the number of protrusions is significantly larger than in the undamaged targets. The protrusions do not collapse during the cutting process with the FIB, which means that they are plastically deformed.

The NRA results for the low- and high-fluence samples show significant retention at $0.5-2 \mu \mathrm{m}$ depth. This depth range correlates well with the locations at which the cavities associated with the blisters were observed. It therefore seems that a significant part of the deuterium is trapped in and/or close to the cavities. Comparison of the surface morphology and the TDS results (figure 7) suggests that the desorption peak at $700 \mathrm{~K}$ is related to the plasma-induced modifications. Using TMAP7 and assuming single traps, this peak was assigned to a trapping energy in the range of $1.4-1.6 \mathrm{eV}$. This energy is too high for the release of deuterium from dislocations $(\sim 0.85 \mathrm{eV}[43$, 44] $)$ and too low for deuterium chemisorbed at the inner surfaces of the cavities to penetrate the grains $(\sim 2.1 \mathrm{eV}[33,45,46])$. After excluding the before mentioned possibilities, we can only speculate about the origin of the $700 \mathrm{~K}$ desorption peak. Obviously, this desorption does not take place via dissociation and chemisorption at the cavity surface (trapping energy of $\sim 2.1 \mathrm{eV}$ ). One possibility would be that gaseous molecular deuterium desorbs via migration along the grain boundaries, i.e. deuterium is released from the cavities via grain boundaries connecting the cavities with the surface area. After all, the growth of the cavities results from pressure build up by accumulation of molecular deuterium. Molecular dynamics simulations by Von Toussaint et al [47] have indeed shown that grain boundaries in polycrystalline tungsten can provide important transport channels. Another option is that during TDS blister bursting occurs [48].

The pre-damaged sample shows in addition a desorption peak at $850 \mathrm{~K}$. This peak has been related to deuterium escaping from vacancy clusters created by the preirradiation with the $\mathrm{MeV}$ ions [36, 41, 49]. These small vacancy clusters are located inside the grains.

The deuterium depth profiles of all samples show a large surface concentration of deuterium $(0-100 \mathrm{~nm}$ depth). This is much more than can be explained by a monolayer of chemisorbed deuterium at the surface $\left(2 \times 10^{19} \mathrm{~m}^{-2}\right)$. In addition, it is likely that molecular deuterium will form. Deuterium molecules have a very low binding to the surface and are likely to desorb. Therefore, we think that the surface peak is a result of the deuterium trapped slightly deeper in the material. Nanostructures and 
protrusions are both likely candidates, although the NRA resolution is not good enough to distinguish between the two.

\section{Conclusions}

In the present experiments, three types of surface modifications were distinguished: inter-granular blisters, intra-granular protrusions and nanostructured surface morphologies. The surface modifications are present well beyond the implantation region. Since the implantation energy is far below the displacement damage threshold, the driving mechanism is most probably super-saturation of the tungsten lattice and subsequent pressure build up at nucleation points. Blisters have been assigned to inter-granular cavities that plastically deform full grains and protrusions to intra-granular cavities that lead to plastic deformation of parts of grains. The detailed mechanisms for the formation of the nanostructures are not clear.

Based on the correlation between depth distributions of cavities and retained deuterium, it seems that a significant part of the deuterium is trapped in and/or close to the cavities. The release of deuterium related to this is observed in the TDS spectrum by means of a desorption peak at $700 \mathrm{~K}$. This may be related to transport of deuterium via grain boundaries or to the bursting of blisters during TDS. Further research is needed to study these results in more detail.

Between the experiments carried out at different plasma flux, large differences were observed. In the low-flux case, i.e. large exposure time, the deuterium penetrated much deeper in the material. This resulted in very large blisters in specific areas on the surface, probably related to weak grain boundaries in the material. High-flux exposure leads to many more but significantly smaller blisters as well as to protrusion formation.

The present results clearly show that experiments carried out at different flux but with the same fluence yield very different results. This is important to consider in cases where extrapolations are made from low-flux experiments to conditions as present in the ITER divertor.

\section{Acknowledgments}

Part of this work, supported by the European Communities under the contract of Association between EURATOM/FOM, was carried out within the framework of the European Fusion Programme with financial support from NWO. The views and opinions expressed herein do not necessarily reflect those of the European Commission. Part of this project has received funding from the Euratom research and training programme 2014-2018.

The authors would like to thank R.S. Al for the technical assistance at the PilotPSI measurements and J. Dorner and M. Fußeder (IPP-Garching) for their technical assistance with the ${ }^{3} \mathrm{He}$ beam analysis. 


\section{References}

[1] $13^{\text {th }}$ ITER Council, 20-21 November 2013.

[2] G. Federici, C. H. Skinner, J. N. Brooks, J. P. Coad, C. Grisolia, A. A. Haasz, A. Hassanein, V. Philipps, C. S. Pitcher, J. Roth, W. R. Wampler, and D. G. Whyte. Plasma-material interactions in current tokamaks and their implications for next step fusion reactors. Nucl. Fusion, 41(12):1967-2137, 2001.

[3] A. S. Kukushkin, H. D. Pacher, A. Loarte, V. Komarov, V. Kotov, M. Merola, G. W. Pacher, and D. Reiter. Analysis of performance of the optimized divertor in ITER. Nucl. Fusion, 49(7):075008, 2009.

[4] J. Roth, E. Tsitrone, A. Loarte, Th. Loarer, G. Counsell, R. Neu, V. Philipps, S. Brezinsek, M. Lehnen, P. Coad, Ch. Grisolia, K. Schmid, K. Krieger, A. Kallenbach, B. Lipschultz, R. Doerner, R. A. Causey, V. Alimov, W. M. Shu, O. V. Ogorodnikova, A. Kirschner, G. Federici, and A. Kukushkin. Recent analysis of key plasma wall interactions issues for ITER. J. Nucl. Mater., 390-391:1-9, 2009.

[5] K. Tokunaga, M. J. Baldwin, R. P. Doerner, N. Noda, Y. Kubota, N. Yoshida, T. Sogabe, T. Kato, and B. Schedler. Blister formation and deuterium retention on tungsten exposed to low energy and high flux deuterium plasma. J. Nucl. Mater., 337-339:887-891, 2005.

[6] V. Kh. Alimov and J. Roth. Hydrogen isotope retention in plasma-facing materials: review of recent experimental results. Phys. Scr., 2007(T128):6, 2007.

[7] W. M. Shu. High-dome blisters formed by deuterium-induced local superplasticity. Appl. Phys. Lett., 92(21):211904, 2008.

[8] W. R. Wampler and R. P. Doerner. The influence of displacement damage on deuterium retention in tungsten exposed to plasma. Nucl. Fusion, 49(11):115023, 2009.

[9] R. D. Kolasinski, D. F. Cowgill, D. C. Donovan, M. Shimada, and W. R. Wampler. Mechanisms of gas precipitation in plasma-exposed tungsten. J. Nucl. Mater., 438(0):S1019-S1022, 2013.

[10] M. Balden, S. Lindig, A. Manhard, and J. H. You. $\mathrm{D}_{2}$ gas-filled blisters on deuterium-bombarded tungsten. J. Nucl. Mater., 414(1):69-72, 2011.

[11] J. H. You. Mechanics of tungsten blistering: A finite element study. J. Nucl. Mater., 437(1-3):2428, 2013.

[12] R. A. Causey. Hydrogen isotope retention and recycling in fusion reactor plasma-facing components. J. Nucl. Mater., 300(2-3):91-117, 2002.

[13] G. N. Luo, K. Umstadter, W. M. Shu, W. Wampler, and G. H. Lu. Behavior of tungsten with exposure to deuterium plasmas. Nucl. Instrum. Methods Phys. Res. B, 267(18):3041-3045, 2009.

[14] B. Tyburska, V. Kh. Alimov, O. V. Ogorodnikova, K. Schmid, and K. Ertl. Deuterium retention in self-damaged tungsten. J. Nucl. Mater., 395(1-3):150-155, 2009.

[15] G. M. Wright, M. Mayer, K. Ertl, G. de Saint-Aubin, and J. Rapp. Hydrogenic retention in irradiated tungsten exposed to high-flux plasma. Nucl. Fusion, 50(7):075006, 2010.

[16] M. Shimada, G. Cao, Y. Hatano, T. Oda, Y. Oya, M. Hara, and P. Calderoni. The deuterium depth profile in neutron-irradiated tungsten exposed to plasma. Phys. Scr., 2011(T145):014051, 2011.

[17] M. H. J. 't Hoen, B. Tyburska-Pueschel, K. Ertl, M. Mayer, J. Rapp, A. W. Kleyn, and P. A. Zeijlmans van Emmichoven. Saturation of deuterium retention in self-damaged tungsten exposed to high-flux plasmas. Nucl. Fusion, 52(2):023008, 2012.

[18] A. Manhard, G. Matern, and M. Balden. A step-by-step analysis of the polishing process for tungsten specimens. Practical Metallography, 50(1):5,16, 2013.

[19] J. F. Ziegler, M. D. Ziegler, and J. P. Biersack. SRIM-the stopping and range of ions in matter. Nucl. Instrum. Methods Phys. Res. B, 268(11-12):1818-1823, 2010.

[20] ASTM E521-96. Standard Practice for Neutron Radiation Damage Simulation by Charge-Particle Irradiation, Annual Book of ASTM Standards, Vol. 12.02. American Society for Testing and Materials, Philadelphia, 1996. 
[21] A. Manhard, T. Schwarz-Selinger, and W. Jacob. Quantification of the deuterium ion fluxes from a plasma source. Plasma Sources Sci. Technol., 20:015010, 2011.

[22] G. J. van Rooij, V. P. Veremiyenko, W. J. Goedheer, B. de Groot, A. W. Kleyn, P. H. M. Smeets, T.W. Versloot, D. G. Whyte, R. Engeln, D. C. Schram, and N. J. Lopes Cardozo. Extreme hydrogen plasma densities achieved in a linear plasma generator. Appl. Phys. Lett., 90(12):121501, 2007.

[23] A. E. Shumack, H. J. de Blank, J. Westerhout, and G. J. van Rooij. Two-dimensional electric current effects on a magnetized plasma in contact with a surface. Plasma Phys. Controlled Fusion, 54(12):125006, 2012.

[24] R. C. Wieggers, D. P. Coster, P. W. C. Groen, H. J. de Blank, and W. J. Goedheer. B2.5-Eunomia simulations of Pilot-PSI plasmas. J. Nucl. Mater., 438(0):S643-S646, 2013.

[25] H. J. van der Meiden, R. S. Al, C. J. Barth, A. J. H. Donne, R. Engeln, W. J. Goedheer, B. de Groot, A. W. Kleyn, W. R. Koppers, N. J. Lopes Cardozo, M. J. van de Pol, P. R. Prins, D. C. Schram, A. E. Shumack, P. H. M. Smeets, W. A. J. Vijvers, J. Westerhout, G. M. Wright, and G. J. van Rooij. High sensitivity imaging Thomson scattering for low temperature plasma. Rev. Sci. Instrum., 79(1):013505, 2008.

[26] D. Bohm. The Characteristics of Electrical Discharges in Magnetic Fields, chapter 3. ed A. Guthrie and R. K. Wakerling (NewYork: McGraw-Hill), 1949.

[27] M. Balden, A. Manhard, and S. Elgeti. Deuterium retention and morphological modifications of the surface of five tungsten grades after deuterium plasma exposure. submitted to J. Nucl. Mater., 2014.

[28] V. Kh. Alimov, M. Mayer, and J. Roth. Differential cross-section of the $\mathrm{D}\left({ }^{3} \mathrm{He}, \mathrm{p}\right){ }^{4} \mathrm{He}$ nuclear reaction and depth profiling of deuterium up to large depths. Nucl. Instrum. Methods Phys. Res. B, 234(3):169-175, 2005.

[29] M. Mayer, E. Gauthier, K. Sugiyama, and U. von Toussaint. Quantitative depth profiling of deuterium up to very large depths. Nucl. Instrum. Methods Phys. Res. B, 267(3):506-512, 2009 .

[30] K. Schmid and U. von Toussaint. Statistically sound evaluation of trace element depth profiles by ion beam analysis. Nucl. Instrum. Methods Phys. Res. B, 281(0):64-71, 2012.

[31] B. M. U. Scherzer. Sputtering by Particle Bombardment II. Springer, Berlin, 1983.

[32] J. B. Condon and T. Schober. Hydrogen bubbles in metals. J. Nucl. Mater., 207(0):1-24, 1993.

[33] M. Poon, A. A. Haasz, and J. W. Davis. Modelling deuterium release during thermal desorption of $\mathrm{D}^{+}$-irradiated tungsten. J. Nucl. Mater., 374(3):390-402, 2008.

[34] D. Nishijima, H. Iwakiri, K. Amano, M. Y. Ye, N. Ohno, K. Tokunaga, N. Yoshida, and S. Takamura. Suppression of blister formation and deuterium retention on tungsten surface due to mechanical polishing and helium pre-exposure. Nucl. Fusion, 45(7):669, 2005.

[35] G. R. Longhurst. TMAP7 user manual. Technical report, INEEL/EXT-04-02352 (Idaho: Idaho National Engineering and Environmental Laboratory), 2004.

[36] M. H. J. 't Hoen, M. Mayer, A. W. Kleyn, H. Schut, and P. A. Zeijlmans van Emmichoven. Reduced deuterium retention in self-damaged tungsten exposed to high-flux plasmas at high surface temperatures. Nucl. Fusion, 53(4):043003, 2013.

[37] H. Eleveld and A. van Veen. Void growth and thermal desorption of deuterium from voids in tungsten. J. Nucl. Mater., 212-215:1421-1425, 1994.

[38] H. Y. Xu, Y.B. Zhang, Y. Yuan, B. Q. Fu, A. Godfrey, G. C. De Temmerman, W. Liu, and $\mathrm{X}$. Huang. Observations of orientation dependence of surface morphology in tungsten implanted by low energy and high flux D plasma. J. Nucl. Mater., 443(1-3):452-457, 2013.

[39] H. K. Birnbaum and P. Sofronis. Hydrogen-enhanced localized plasticity - a mechanism for hydrogen-related fracture. Materials Science and Engineering: A, 176(12):191-202, 1994.

[40] Y. Liang, P. Sofronis, and R. H. Dodds Jr. Interaction of hydrogen with crack-tip plasticity: effects of constraint on void growth. Materials Science and Engineering: A, 366(2):397 - 411, 2004 . 
[41] A. van Veen, H. A. Filius, J. De Vries, K. R. Bijkerk, G. J. Rozing, and D. Segers. Hydrogen exchange with voids in tungsten observed with TDS and PA. J. Nucl. Mater., 155-157:11131117, 1988.

[42] E. Markina. Private communication.

[43] H. Eleveld. Hydrogen and helium in selected fusion reactor materials. PhD thesis, TU-D, Delft, the Netherlands, 1996.

[44] O. V. Ogorodnikova, J. Roth, and M. Mayer. Deuterium retention in tungsten in dependence of the surface conditions. J. Nucl. Mater., 313-316:469-477, 2003.

[45] H. Eleveld and A. van Veen. Deuterium interaction with impurities in tungsten studied with TDS. J. Nucl. Mater., 191-194(Part 1):433-438, 1992.

[46] M. Fukumoto, H. Kashiwagi, Y. Ohtsuka, Y. Ueda, M. Taniguchi, T. Inoue, K. Sakamoto, J. Yagyu, T. Arai, I. Takagi, and T. Kawamura. Deuterium trapping in tungsten damaged by high-energy hydrogen ion irradiation. J. Nucl. Mater., 390-391:572-575, 2009.

[47] U. von Toussaint, S. Gori, A. Manhard, T. Höschen, and C. Höschen. Molecular dynamics study of grain boundary diffusion of hydrogen in tungsten. Phys. Scr., 2011(T145):014036, 2011.

[48] A. Manhard, U. von Toussaint, T. Dürbeck, K. Schmid, and W. Jacob. Statistical analysis of blister bursts during temperature-programmed desorption of deuterium-implanted polycrystalline tungsten. Phys. Scr., (T145):014038, 2011.

[49] M. Poon. Deuterium trapping in tungsten. PhD thesis, University of Toronto, Canada, 2004. 